\section{IDENTIFICATION OF MICRORNA PREDICTIVE OF TREATMENT RESPONSE IN LUPUS NEPHRITIS}

${ }^{1} \mathrm{~S}$ Hasni ${ }^{*},{ }^{2} \mathrm{M}$ Hadavand, ${ }^{3} \mathrm{H}$ Zhou, ${ }^{2} \mathrm{~N}$ Binmadi, ${ }^{2} \mathrm{M}$ Tandon, ${ }^{2}$ I Alevizos. ${ }^{1}$ National Institutes of Health, National Institute of Arthritis and Musculoskeletal and skin diseases, Bethesda, USA; ${ }^{2}$ National Institutes of Health, National Institute of Dental and Craniofacial research, Bethesda, USA; ${ }^{3}$ National Institutes of Health, National Institute of Dental and Craniofacial Research NIDCR, Bethesda, USA

\subsection{6/lupus-2017-000215.275}

Background and aims High dose corticosteroids and cyclophosphamide are commonly used to treat LN. Although effective in preventing end stage renal disease (ESRD) in most cases, significant long-term side effects such as infections, increased risk of malignancy, and infertility are common and are related to the duration of therapy or the cumulative dose of medications. There are currently no markers that can reliably determine response or refractoriness to treatment at an individual level. MicroRNAs are small, non-coding RNAs responsible for post-transcriptional regulation, have been shown to have altered expression levels in a variety of diseases suggesting their potential use as biomarkers. We propose miRNAs can be predictive markers for response to cyclophosphamide.

Methods RNA was isolated and analysed via TaqMan Array MicroRNA 384 well Cards, from formalin-fixed paraffin embedded (FFPE) renal biopsies of two cohorts of patients with LN who were subsequently treated with cyclophosphamide with at least 2 years of follow up history. Patients who responded to cyclophosphamide based on urinalysis criteria of no active urinary sediments, no RBCs or WBCs in urine, and proteinuria less than 1 gram were classified as responders while those that did not fit the criteria were classified as nonresponders. Significantly deferentially expressed miRNAs, determined via $2^{\Delta \Delta \mathrm{Ct}}$ method, from the first cohort were validated by the second cohort.

Results Six significantly up-regulated miRNAs, hsa-miR-30c-23p, hsa-miR-29b-1-5p, hsa-miR-195-3p, hsa-miR-424-3p, hsamiR-1260a, and hsa-miR-1248 were found in responders.

Conclusions These miRNAs may act as prognostic markers of renal outcomes and treatment response, which can establish a more personalised treatment of lupus nephritis in the future.

\section{LOW-DOSE IL-2 CIRCUMVENTED MTOR SIGNALLINGSIGNALING IN T CELLS IN THE TREATMENT OF SLE}

1J He, 'X Sun, 'J Li, 'R Zhang*, 'D Yu, 'Z Li. 'Peking Univ. People's Hospital, Rheumatology and Immunology, Beijing, China; ${ }^{2}$ School of Biomedical Sciences, ImmunologyClayton, Victoria, Australia

\subsection{6/lupus-2017-000215.276}

Background and aims mTOR signalling is proved to be one of the most important pathway in the pathogenesis in SLE. However, in patients with SLE, whether mTOR pathway can be activated by low-dose IL-2 remained unclear.This study is to clarify the effects of low-dose IL-2 therapy on mTOR signalling in the treatment of SLE.

Methods Eight patients with active SLE were treated with 1 million IU IL-2. Phophrylation of S6 ribosomal protein
(S6RP), AKT and pSTAT5 were measured before and after the first 2 week of low-dose rhIL-2 administration. C57BL/6 mice (male, 8-12 weeks old) were intraperitoneally immunised with $\mathrm{SRB}$ and followed by administration of different doses (low:10 000 IU and hight:300000 IU) of rhIL-2 or PBS from day 3 to day 9. The ratio of Th1, Th2, Tfh, Th17, Tfh and Treg as well as the level of S6RP, AKT and pSTAT5 were assayed by flow cytometry.

Results Low-dose IL-2 was efficient and well tolerated in active SLE, and was associated with expansion of Treg cells $(p<0.001)$ and reductions of Tfh and Th17 cells $(p=<0.001)$. No significant change of pS6RP and pAKT was observed. On the other hand, there was a signfciant induction of the activation of STAT5. In mouse studies, low-dose IL-2 inhibited the differentiation of Th17 cells and Tfh cells. Comparing with high dose IL-2 group, there was no significantly increased mTOR activity after low-dose IL-2 administration.

Conclusions Low-dose IL-2 might circumvent mTOR pathway and play a regulatory role in the $\mathrm{T}$ cells in lupus.

\section{USEFULNESS OF SOLUBLE PD-1 IN PATIENTS WITH SYSTEMIC LUPUS ERYTHEMATOSUS}

S Hirahara*, Y Katsumata, Y Kawaguchi, H Yamanaka. Tokyo Women's Medical University, Institute of Rheumatology, Tokyo, Japan

\subsection{6/lupus-2017-000215.277}

Background and aims Programmed cell death protein 1 (PD-1/ CD279) is a cell surface receptor that belongs to the extended CD28/CTLA-4 family and is expressed on $\mathrm{T}$ cells and pro-B cells. PD-1 plays an important role in down regulating the immune system by preventing the activation of T-cells. Soluble PD-1 (sPD-1), which is produced by the alternative splicing, can functionally block the regulatory effect of membranebound PD-1 on $\mathrm{T}$ cell activation. We aimed to retrospectively evaluate the usefulness of sPD-1 in patients with systemic lupus erythematosus (SLE).

Methods We measured the levels of sPD-1 by enzyme-linked immunosorbent assay (ELISA) kit in sera of patients with SLE $(n=59)$ and systemic sclerosis, and healthy controls, and compared them. We also analysed the association between the levels of sPD-1 and clinical information in patients with SLE.

Results The levels of sPD-1 in SLE patients with SLEDAI$2 \mathrm{~K} \geq 6$ were significantly higher than those in SLE patients with SLEDAI- $2 \mathrm{~K}<6$, patients with systemic sclerosis, and healthy controls $(\mathrm{p}<0.05$ in all comparisons), whereas there was no significant difference in other comparisons. In SLE patients, the levels of sPD-1 were moderately correlated with the titers of anti-ds DNA antibodies and SLEDAI-2K, and were moderately and inversely correlated with the levels of C3 and C4. In addition, the levels of sPD-1 were significantly higher in SLE patients with arthritis, mucosal ulcers, fever, leucopenia, or anaemia than those without $(p<0.05$ in all comparisons).

Conclusions The present study suggested that sPD-1 can serve as a new biomarker reflecting disease activity in patients with SLE. 